\title{
Retrograde cerebral perfusion during hypothermic circulatory arrest reduces neurologic morbidity
}

Hypothermic circulatory arrest has become an accepted technique for a variety of cardiac and complex aortic operations. However, prolonged periods ( $>45 \mathrm{~min}$ ) of hypothermic circulatory arrest in older patients is associated with marginal cerebral protection and an increased incidence of adverse neurologic events. In an effort to minimize such morbidity, we used a technique of retrograde cerebral perfusion with continuous monitoring of cerebral hemoglobin oxygen saturation during hypothermic circulatory arrest in 35 patients who underwent thoracic aortic operations or resection of intracardiac tumor. There were 27 men and 8 women (mean age 60 years, range 21 to 83 years). Sixteen patients had acute dissection, 6 had contained rupture of a thoracic aortic aneurysm, 10 had either a chronic dissection or aneurysm, and 3 had hypernephromas extending into the heart. Six patients underwent root replacement by means of an open technique for their distal anastomosis, 7 underwent root and partial arch replacement, 12 had root and total arch replacement, 7 had total arch replacement, and 3 had resection of tumor in the heart and retrohepatic vena cava. Seven patients had simultaneous coronary artery bypass grafting, 3 had replacement of one of the arch vessels, and 2 patients had a cesarean section. Sixteen cases were emergency, 6 urgent, and 13 elective. Nine $(26 \%)$ were reoperations. Thirty-four patients underwent the procedure via a median sternotomy and one patient through a posterolateral thoracotomy. The mean retrograde cerebral perfusion time was 63 minutes (range 35 to 128 minutes), with $30(86 \%)$ patients having more than 45 minutes, $12(34 \%)$ having more than 65 minutes, and $4(11 \%)$ having more than 90 minutes. There was 1 operative death caused by a preoperative myocardial infarction from an aortic dissection, and there were 2 late deaths (multiple organ failure and ruptured total aortic aneurysm). One patient had a stroke with a residual right hemiplegia and a pronounced aphasia. There were no other significant neurologic events or reoperations for bleeding. The average length of stay for patients having elective operations was 11 days and for those having emergency operations, 27 days. At a mean follow-up of 6 months all surviving patients (91\%) are well. Hypothermic circulatory arrest is a relatively simple technique that provides a bloodless field and good visualization without the need for aortic crossclamps. Moreover, retrograde cerebral perfusion with continuous monitoring of cerebral oxygen saturation extends the "safe" time for hypothermic circulatory arrest, allowing ample opportunity to perform complicated cardiac and aortic operations with reduced risk of adverse neurologic events. (J THORAC CARDIOvASC SURG 1995;109:259-68)

\section{G. Michael Deeb, MD, Eric Jenkins, CCT, CCP (by invitation), Steven F. Bolling, MD,}

Louis A. Brunsting, MD (by invitation), David M. Williams, MD (by invitation),

Leslie E. Quint, MD (by invitation), and Nancy D. Deeb, RN (by invitation), Ann Arbor, Mich.

From the University of Michigan Medical Center, Section of Thoracic Surgery, Perfusion Services, and Department of Radiology, Ann Arbor, Mich.

Read at the Seventy-fourth Annual Meeting of The American Association for Thoracic Surgery, New York, N.Y., April 24-27, 1994.

Address for reprints: G. Michael Deeb, MD, 1500 E. Medical Center Dr., Box 0344, Ann Arbor, MI 48109.

Copyright (C) 1995 by Mosby-Year Book, Inc.

$0022-5223 / 95 \$ 3.00+0 \quad \mathbf{1 2 / 6 / 6 1 1 3 4}$
A lthough Michael DeBakey and his associates ${ }^{1}$ reported the first successful proximal aortic arch replacement in 1957 , uncontrollable perioperative bleeding and central nervous system damage have accounted for the high perioperative mortality and morbidity of subsequent reported cases. ${ }^{2,3}$ The successful use of profound hypothermia and circulatory arrest (HCA) for infants with complex congenital 


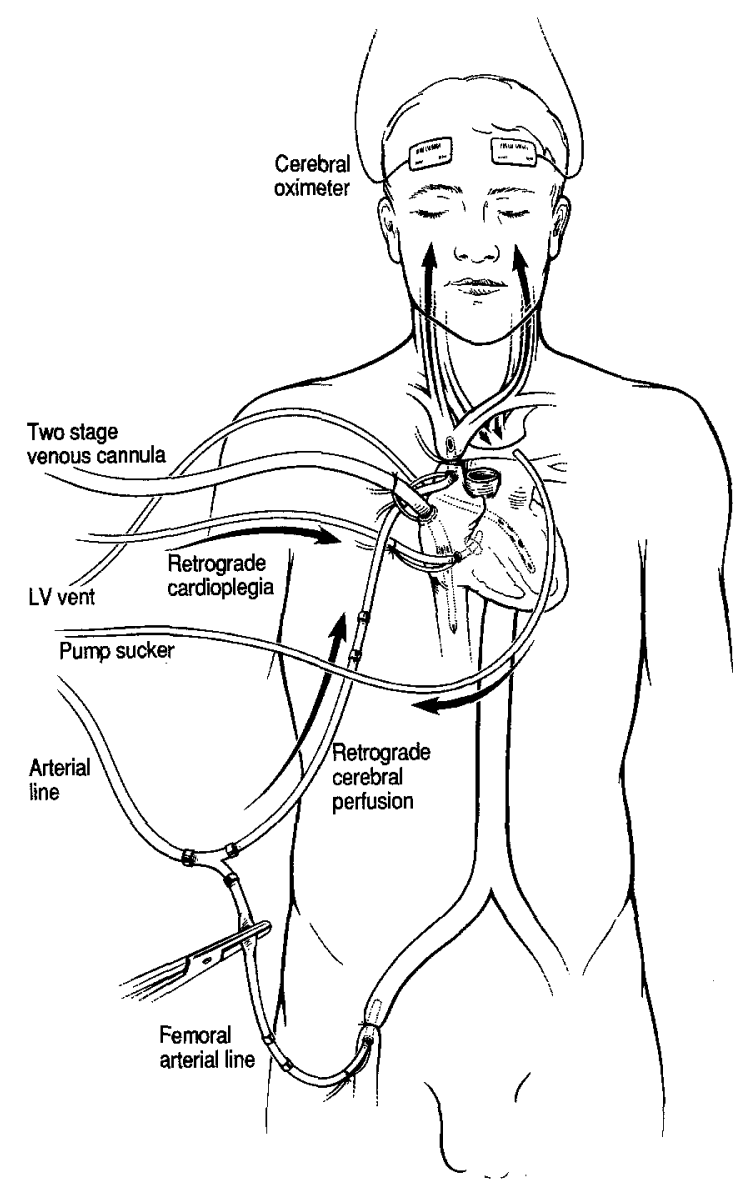

Fig. 1. Schema for insertion of intravenous cannulas and placement of spectroscopy patches with the patient supine. $L V$, Left ventricular.

heart anomalies encouraged Griepp and colleagues ${ }^{4}$ to use this method for repair of aortic arch aneurysms. Even though cardiopulmonary bypass, profound hypothermia, and circulatory arrest became well established in the surgical treatment of arch aneurysms, ${ }^{5-9}$ Svensson and coworkers ${ }^{10}$ showed that cerebral ischemic times greater than 45 minutes were associated with a higher risk of stroke, and ischemic times greater than 65 minutes were associated with a higher mortality. In an effort to allow adequate time for complete and precise repair of aortic arch lesions, as well as to avoid air embolism, Ueda and associates ${ }^{11}$ introduced first intermittent and later continuous retrograde cerebral perfusion (RCP) via the superior vena cava as a adjunct to HCA. Safi and colleagues ${ }^{12}$ reported a prospective series to define the role of RCP during HCA for the repair of aortic arch aneurysms.

From January 1991 to January 1993, seven pa- tients at our institution underwent repair of aortic dissection or arch aneurysm with HCA as adjuvant therapy. Four patients whose HCA times were less than 60 minutes had no neurologic deficit as noted by basic neurologic examination. However, three patients whose HCA times were greater than 60 minutes (67, 72, and 109 minutes, respectively) never regained meaningful consciousness and died. Encouraged by the report of Ueda's group, ${ }^{11}$ we incorporated the technique of RCP in association with HCA in a concerted effort to decrease neurologic complications and improve the outcome of patients undergoing operations for aortic dissection or arch aneurysm repair. This report describes our experience using RCP in conjunction with HCA for the repair of complex cardiovascular lesions and introduces the concept of monitoring continuous cerebral hemoglobin oxygen saturation as an indicator of cerebral oxygen delivery during these procedures.

\section{Patients and methods}

From January 1993 to March 1994, 35 patients underwent repair of complex cardiovascular lesions with HCA and RCP. There were 27 male and 8 female patients. Ages ranged from 21 to 83 years with a mean age of 60 years. There were 16 emergency operations for acute dissection of the aorta, 6 urgent operations for contained rupture of a thoracic aortic aneurysm, 10 elective operations for either a chronic aortic aneurysm or dissection, and 3 elective operations for renal cell carcinoma with extension into the right atrium. Seven patients had simultaneous coronary artery bypass procedures and 2 patients with acute dissection underwent cesarean section. Nine procedures $(26 \%)$ were re-operative. In 34 patients the approach was through a median sternotomy and in 1 patient via a posterolateral thoracotomy.

Positioning and access. Patients undergoing a median sternotomy were placed supine, and access for cardiopulmonary bypass (CPB) was obtained via the femoral artery and a two-stage cannula in the right atrium. The left ventricle was decompressed with a vent placed through the right superior pulmonary vein. A cardioplegia catheter was placed in the coronary sinus for retrograde cardioplegia. The arterial line was bifurcated and a long rightangled cannula was placed high in the superior vena cava for RCP. The positioning of the tip of this cannula may be critical because isolated reports have been published of a valve at the confluence of the superior vena cava and the internal jugular vein that could inhibit retrograde flow if the tip of the cannula were not positioned beyond this point. Somanetics spectroscopy patches (Somanetics Corp., Troy, Mich.) were placed over both cerebral hemispheres to monitor the cerebral oxygen saturation (Fig. 1).

When the posterolateral approach was used, access to the left femoral artery and vein, as well as the internal 
jugular vein, was obtained with the patient in a supine position. Vessel loops were placed around the artery and veins and the skin was closed. The patient was then placed in a lateral position, reprepared, draped, and the thorax was entered through a posterolateral incision in the fourth intercoastal space. The cervical and femoral incisions were reopened, and CPB was accomplished by way of the femoral artery and a long venous catheter threaded over a guide wire through the femoral vein and positioned in the right atrium with the aid of intraoperative transesophageal echocardiography. The venous line was bifurcated and a right-angled cannula was placed in the left atrium and positioned across the mitral valve for complete drainage and decompression of the left ventricle. The arterial line was bifurcated and a small right-angled cannula was placed in the internal jugular vein for RCP. The Somanetics spectroscopy patches were placed over both cerebral hemispheres (Fig. 2).

CPB circuit. The CPB circuit used an Avecor silicone membrane lung (Avecor Cardiovascular Inc., Minneapolis, Minn.) and a Minntech HP600 hemoconcentrator (Minntech Corp., Minneapolis, Minn.) (Fig. 3). If the pre-CPB hematocrit value was greater than $30 \%, 1$ unit of whole blood was removed and replaced with colloid solution. If the hematocrit value was greater than $35 \%, 2$ units were removed and replaced with colloid solution. The CPB prime was as follows: $2 \mathrm{~L}$ electrolyte solution (Normosol-R), $500 \mathrm{ml}$ hetastarch (Hespan), $50 \mathrm{gm}$ albumin, 50 gm $\mathrm{NaHCO}_{3}, 25 \mathrm{gm}$ mannitol, 10,000 units heparin, $1 \mathrm{gm}$ methylprednisolone, $1 \mathrm{gm}$ ceftazidime, $1 \mathrm{gm}$ vancomycin (titrated on CPB), and $500,000 \mathrm{kIU}$ aprotinin. The central venous pressure was measured above the tourniquet of the RCP catheter. Nasopharyngeal and bladder temperatures were monitored continuously.

Once CPB was instituted, the patient was cooled to a bladder temperature of $16^{\circ} \mathrm{C}$, with a $8^{\circ}$ to $12^{\circ} \mathrm{C}$ gradient being maintained between the bladder and the perfusate temperature. Cooling time ranged between 60 and 110 minutes depending on the patient's body surface area. When the desired temperature was reached, the patient was placed in the Trendelenburg position, $1 \mathrm{gm}$ thiopental and $25 \mathrm{gm}$ mannitol were given intravenously, and CPB was discontinued. The tourniquet around the RCP catheter was tightened, and the clamp was removed from the $\mathrm{RCP}$ line and placed on the femoral arterial line. RCP flow rates ranged from $500 \mathrm{ml} / \mathrm{min}$ to $0.8 \mathrm{~L} / \mathrm{min} /$ per square meter while maintaining a cerebral oxygen saturation within $10 \%$ of the baseline value and a central venous pressure between 15 and $25 \mathrm{~mm} \mathrm{Hg}$. At the time that CPB was reinstituted, the clamp on the femoral arterial line was removed to allow simultaneous flow with the RCP line to avoid air embolism. When the air was evacuated, the RCP line was clamped and the tourniquet loosened. The patient was rewarmed, the same $8^{\circ}$ to $12^{\circ} \mathrm{C}$ gradient being maintained. Rewarming times ranged between 90 and 150 minutes. During rewarming the volume in the circuit was hemoconcentrated to allow the hematocrit value to rise above $25 \%$.

Continuous monitoring of oxygen saturation. The hemoglobin oxygen saturation in the brain tissue was monitored with spectroscopy instrument (INVO3 2910, Somanetics) (Fig. 4). Near-infrared light is generated with a

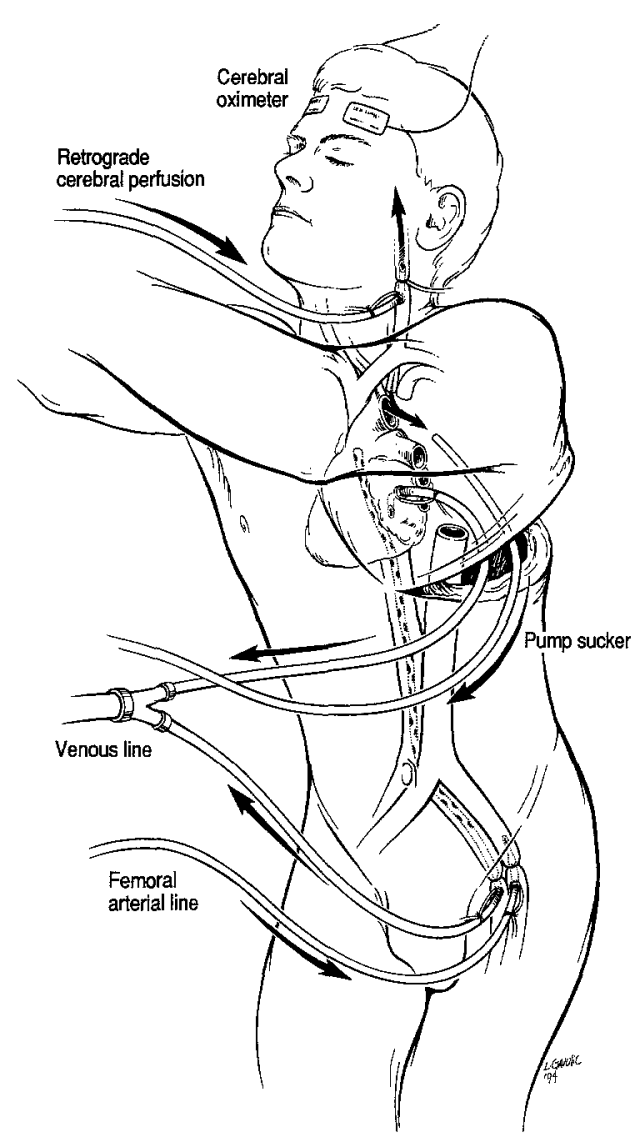

Fig. 2. Schema for insertion of intravenous cannulas and placement of spectroscopy patches for operations performed via the posterolateral approach.

spectrum from 650 to $1100 \mathrm{~nm}$. Cables deliver the light to the patient through a patch placed on the forehead. Light entering the tissue is absorbed or scattered, and portions of it pass back through the surface near the entry point. A single patch on the forchead houses the light source and one or more fiberoptic light guides to capture the returning light. The tips of the sensor light guides are separated from the source by distances calculated to control the mean path of the light passing through the patient. The distance will then determine the depth of the tissue from which the light source is captured.

For scalp and skull tissue to be separated when brain sampling is desired, the shallow part of the field (scalp and skull) must be distinguished from the deep part (brain). This is accomplished by positioning of the two sensors in the patch, one being sufficiently close to the source to sample primarily shallow tissue and the second at an appropriate distance to sample deep tissue. Shallow tissue contribution to attenuation is subtracted from the total attenuation, so that only the attenuation of the deep tissues remains.

The majority of attenuation of the near-infrared light is due to absorption by hemoglobin. Because hemoglobin and oxyhemoglobin have unique absorption profiles, a 


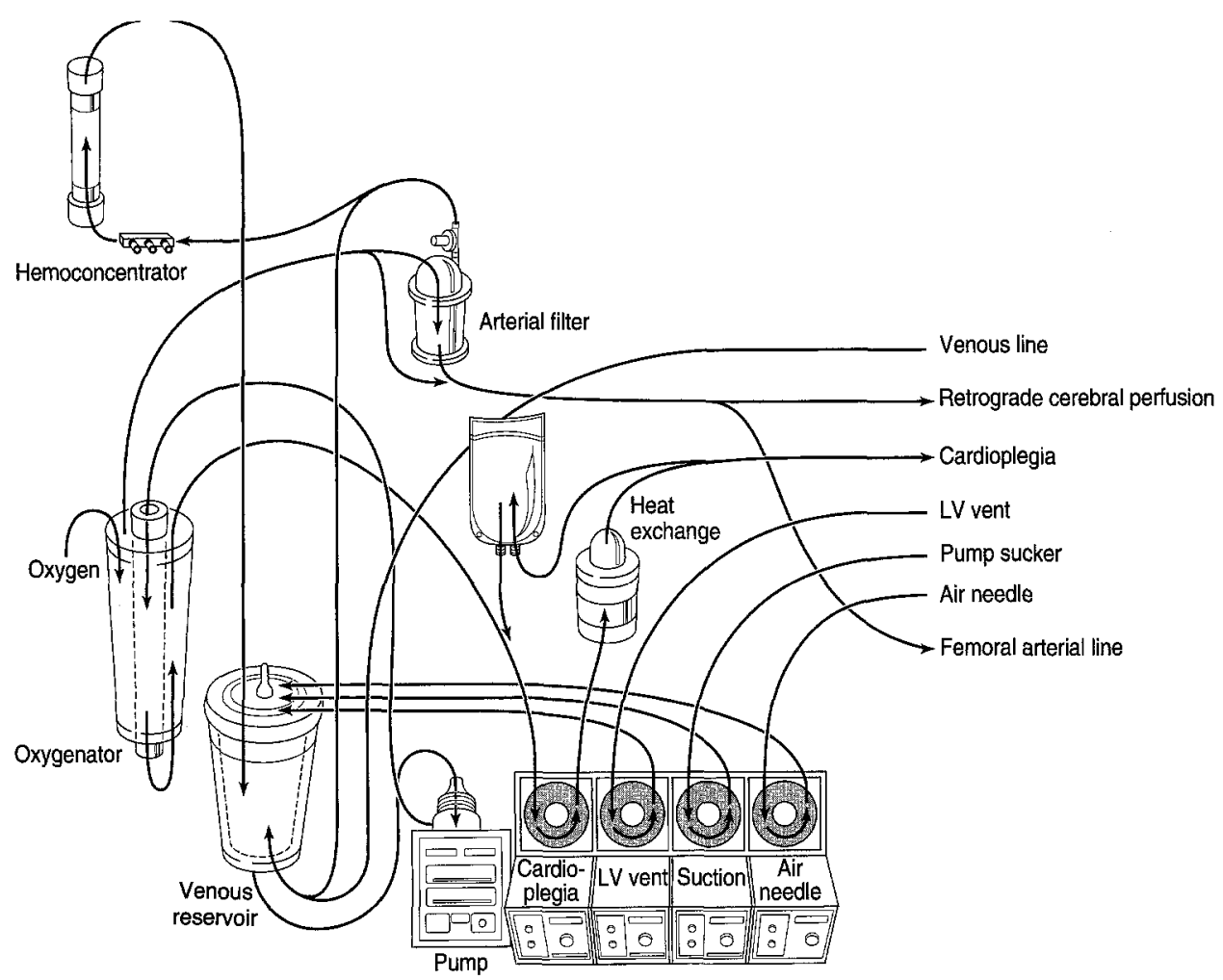

Fig. 3. The CPB circuit. $L V$, Left ventricular.

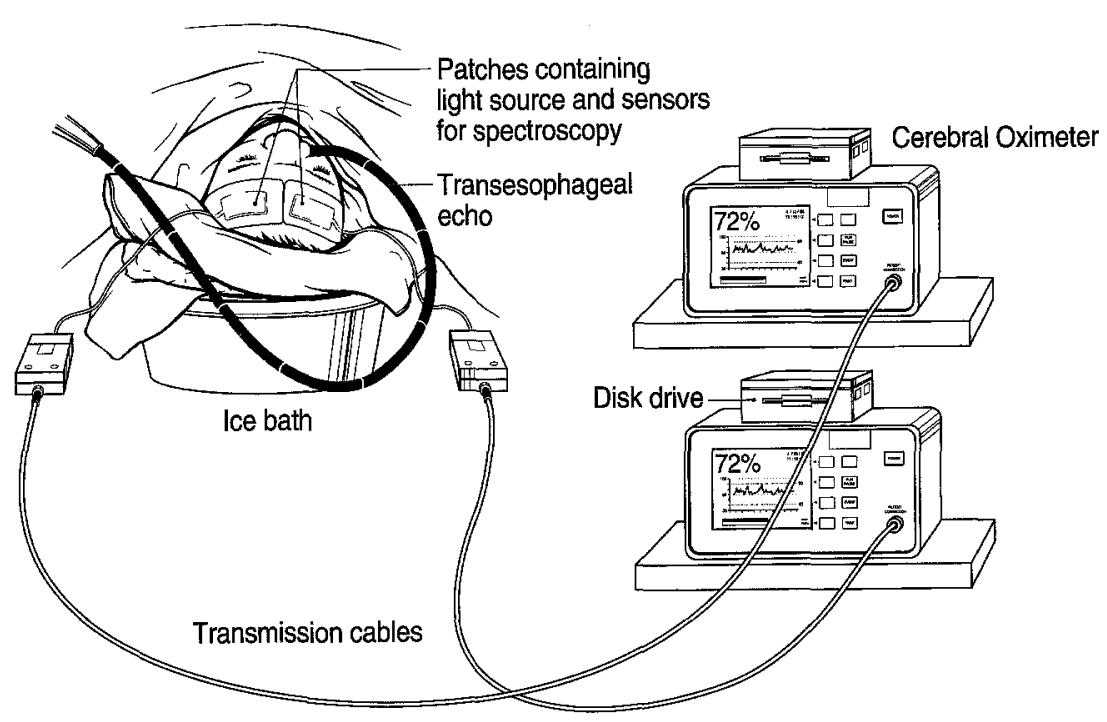

Fig. 4. Spectroscopy instrument used to monitor hemoglobin oxygen saturation in the brain tissue.

ratio of the two can be calculated, and a percent of oxyhemoglobin to total hemoglobin can be expressed as the saturation. ${ }^{13}$ The instrumentation can continually graph percent saturation versus time for a continual, noninvasive, and direct reading. Each patient served as his or her own control with baseline established as the cerebral oxygen saturation before CPB. The RCP was regulated during HCA to maintain cerebral oxygen saturation at $10 \%$ of baseline.

Surgical procedures. Fig. 5 shows the eight different procedures performed on the 35 patients. Six patients with acute dissection and the intimal tear at the sinotubular 
A
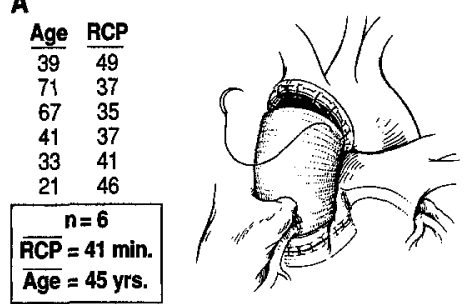

c
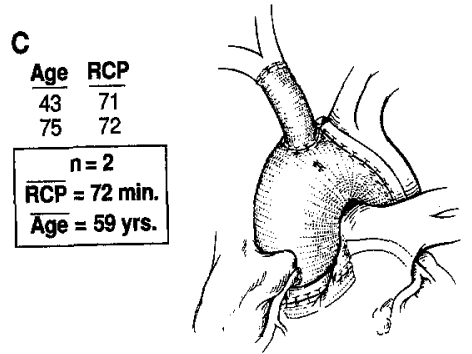

E
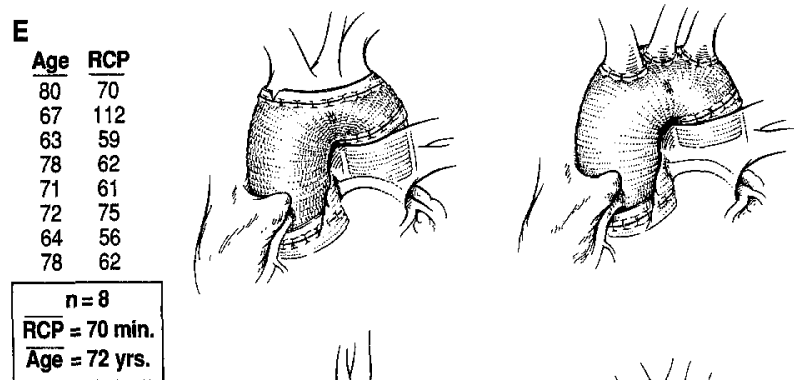
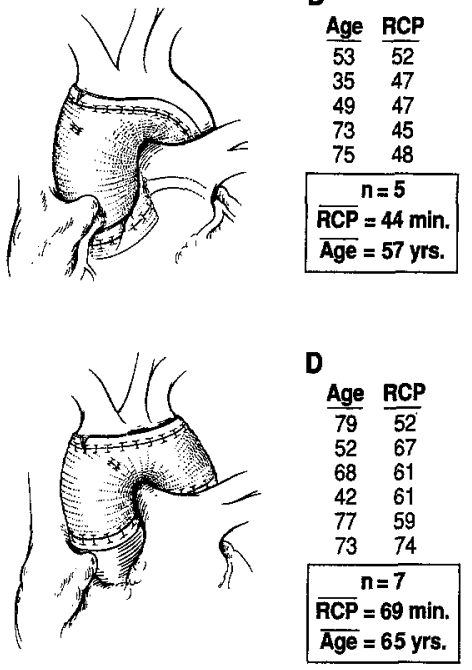

B

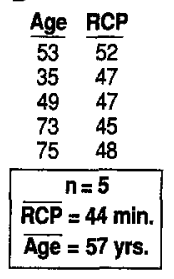

F

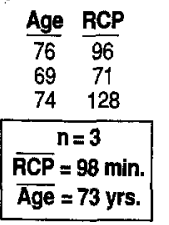

G

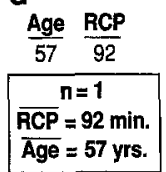

H

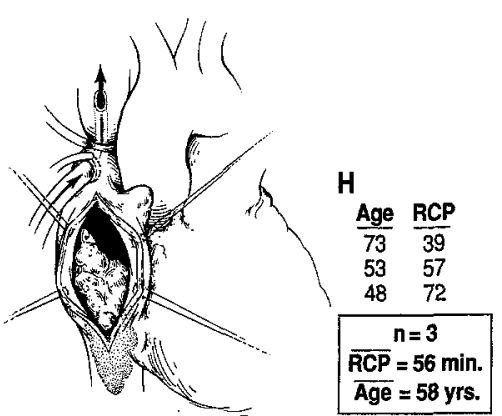

Average RCP Time $=63 \mathrm{~min}$

Average Patient Age $=60 \mathrm{yrs}$.

Fig. 5. Diagram of the eight different procedures performed on the 35 patients. See text for explanation.

ridge underwent root replacement with the distal anastomosis at the level of the innominate artery performed in an open fashion by means of a double-layer felt sandwich technique for reinforcement $(A)$. Five patients underwent root, ascending aorta, and partial arch replacement for aneurysmal disease $(B)$. Two patients underwent the same procedure as $B$, but in addition they had replacement of an aneurysmal innominate artery $(C)$. Seven patients underwent complete arch replacement with reimplantation of the arch vessels as a single island $(D)$. Eight patients had complete root and arch replacement with the elephant trunk technique used as a staged procedure for complete replacement of the aorta $(E)$. Three patients underwent procedure $E$, but because of acute dissection with the tear involving the base of the arch vessels, each arch vessel was implanted separately $(F)$. One patient had procedure $F$, but because of subsequent rupture of the anastomosis of the left common carotid artery to the graft after $\mathrm{CPB}$, the common carotid was replaced without $\mathrm{CPB}$ $(G)$. Three patients underwent removal of renal cell 


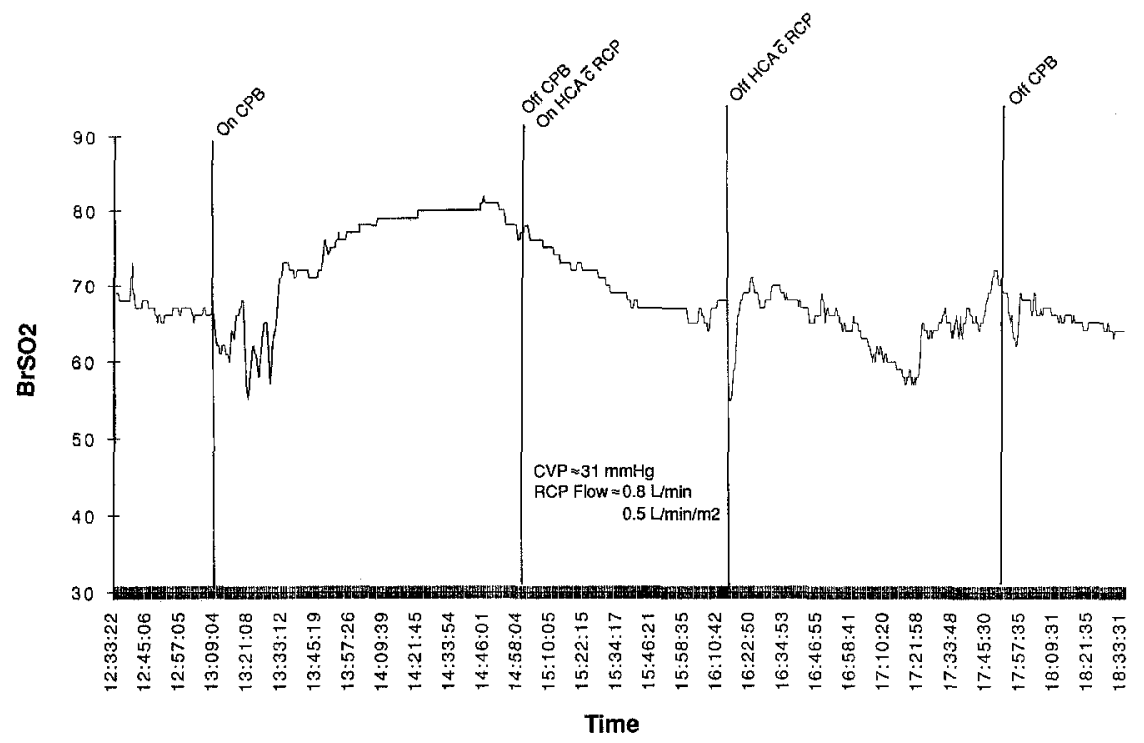

Fig. 6. Brain oxygen saturation ( $\mathrm{BrO} 2$ ) versus time during $\mathrm{HCA}$ and RCP.

carcinoma from the right atrium and inferior vena cava with pericardial patch repair of the subhepatic inferior vena cava $(H)$.

Neurologic assessment. All patients undergoing elective or urgent operations had a standard basic preoperative physical examination and neurologic assessment by the thoracic surgical service. Patients requiring emergency procedures were taken immediately to the operating room, and preoperative assessment was as complete as time and conditions would permit. On extubation and removal of the monitoring lines, all patients underwent the same basic physical examination. The neurologic component was sensitive for gross motor and sensory deficit. No precise neuropsychometric testing was performed on this patient population by a member of the neurosciences department unless an abnormality was noted by physical examination.

\section{Results}

RCP time ranged from 35 to 128 minutes with a mean of 63 minutes. Thirty of the 35 patients $(86 \%)$ had RCP times greater than 45 minutes, $12(34 \%)$ greater than 65 minutes, and $4(11 \%)$ had RCP for periods exceeding 90 minutes. Fig. 5 shows the mean RCP for each of the eight procedures. There was a definite increase in the RCP time with increased complexity of the procedure. Groups A and B, which involve one distal anastomosis, had a mean RCP of 44 minutes; groups $\mathrm{C}, \mathrm{D}$, and $\mathrm{E}$, which involve at least two distal anastomoses, had a mean RCP of 69 minutes; and groups F and G, which had multiple distal anastomoses, had a mean RCP of 97 minutes.

Cerebral oxygen saturation was continually mon- itored during the procedure in an effort to obtain information concerning cerebral oxygen delivery and possible brain ischemia. RCP was regulated to maintain cerebral oxygen saturation within $10 \%$ of baseline. Fig. 6 is a graph of cerebral oxygen saturation versus time in a typical patient undergoing HCA with RCP. Fig. 7 graphically displays the relationship between both antegrade and retrograde flow in conjunction with the cerebral oxygen saturation. As noted, RCP maintained the cerebral oxygen saturation within $10 \%$ of baseline without great fluctuations of flow rate. There was no difference in the cerebral oxygen saturation between the right and left hemispheres in any of the patients who had RCP via the superior vena cava. In the patient who underwent RCP via the left internal jugular vein, the left cerebral oxygen saturation was consistently $10 \%$ to $15 \%$ higher than that of the right side.

Thirty-three patients $(94 \%)$ were awake and following simple commands within 24 hours of the operation. At the time of extubation all were oriented to time, place, and person once reestablished via verbal communication. No deficits to gross motor or sensory function were observed in this group of 33 patients. One patient (3\%) had a neurologic event as documented by computed tomographic scan as a left cerebral infarct, which resulted in a significant hemiplegia on the right side and expressive aphasia ( $G, \mathrm{RCP} 92$ minutes). This patient was the only patient whose extubation was delayed because of neurologic conditions and who required 


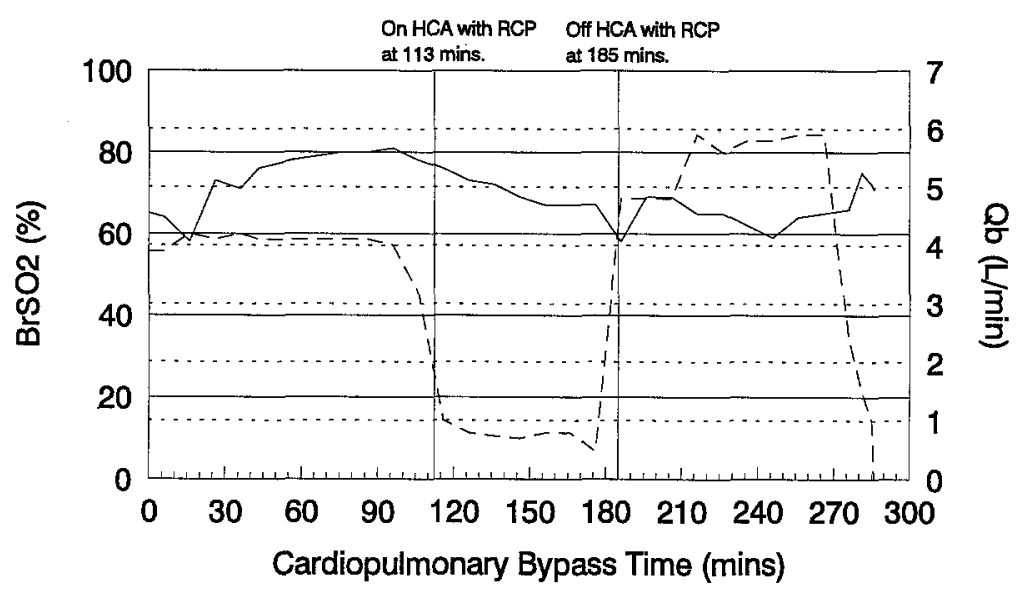

Fig. 7. Relationship between antegrade and retrograde flow $(O b)$ in conjunction with brain oxygen saturation ( $\mathrm{BrO} 2)$ during $\mathrm{CPB}$ and $\mathrm{HCA}$ with RCP for resection of an intravascular renal tumor. Solid line, Brain oxygen saturation; dashed line, relationship between antegrade and retrograde flow.

a tracheostomy. The other 33 patients were extubated on the basis of underlying cardiopulmonary disease.

One perioperative death (3\%) occurred in a patient with an acute dissection and a preoperative myocardial infarction with cardiogenic shock ( $E$ plus four coronary bypass grafts, RCP 61 minutes). In addition, two late deaths occurred (6\%) (greater than 60 days), one from postoperative right heart failure ( $A$ plus one coronary bypass graft, RCP 37 minutes) in a patient who died of multiorgan failure, and one from acute rupture of a descending aortic aneurysm in a patient who was awaiting completion of staged replacement of the entire aorta $(E, \mathrm{RCP}$ 75 minutes). One patient had postoperative mediastinitis necessitating rectus flaps for resolution $(A)$. Multiple pulmonary emboli occurred in one patient despite adequate anticoagulation and necessitated insertion of a Greenfield filter $(D)$. The patient who underwent arch replacement via the posterolateral thoracotomy $(D)$ had a postoperative chylothorax that necessitated transthoracic ligation. Finally, two patients required the insertion of permanent atrioventricular sequential pacemakers for heart block $(D$ and $F)$. No reoperations for bleeding were necessary. Thirty-three $(94 \%)$ of the patients were discharged from the hospital in good health, and 31 $(89 \%)$ are presently alive and well (average follow-up 11 months). Average postoperative stay for the patients having elective operations was 11 days and for those having urgent and emergency operations, 27 days. One of the two infants delivered by cesarean section died in the hospital of respiratory insufficiency and the second infant is alive and well more than 1 year after delivery.

\section{Discussion}

Aortic arch surgery remains a formidable technical challenge that demands precise reconstruction of markedly diseased and friable tissues and sufficient time to perform the procedure meticulously. The introduction by Griepp of HCA was a major contribution to the development of a safe surgical technique that would afford adequate time and a dry motionless field to accomplish the necessary repair. The contributions of Crawford and Cooley, allowing for an open technique without the encumbrance of surgical clamps and the necessity of tedious dissection, hastened the procedure and decreased the associated surgical bleeding. However, Svensson showed in a retrospective review of 656 patients undergoing HCA that there is a distinct time limit ( 45 minutes) before the risk of a neurologic event or death becomes significant. This time limitation is because cerebral oxygen delivery stops whereas metabolism is only reduced. ${ }^{14}$

Hypothermia is known to reduce the neuronal injury associated with hypoxia. ${ }^{15-19}$ This protective mechanism is related to a decrease in metabolic rate in association with falling temperatures and a simultaneous reduction in oxygen requirement by the neurons. The reduction in the metabolic rate can be quantified as the change in metabolism of the tissue for every $10^{\circ} \mathrm{C}$ decrease in temperature $\left(\mathrm{Q}_{10}\right)$ as follows: $\mathrm{Q}_{10}=(\mathrm{R} 1 / \mathrm{R} 2)^{10 /(\mathrm{T} 1-\mathrm{T} 2)}$, where $\mathrm{R} 1$ and $\mathrm{R} 2$ represent the metabolic rate at temperatures $\mathrm{T} 1$ and 
$\mathrm{T} 2$, respectively. ${ }^{20}$ In human beings the metabolic rate is approximately halved with a $10^{\circ} \mathrm{C}$ decrease in temperature $\left(\mathrm{Q}_{10}=2\right)$. Thus cooling from $37^{\circ}$ to $16^{\circ} \mathrm{C}$ during HCA should significantly decrease the metabolic need and the oxygen consumption of the tissue. Because cellular oxygen demand persists during HCA and oxygenated blood is left behind in the microcirculation of the brain, intravascular oxygen should move down its partial pressure $\left(\mathrm{PO}_{2}\right)$ gradient to the mitochondria. Mitochondrial $\mathrm{Po}_{2}$ is 3 to 8 torr, giving an initial gradient of greater than 80 torr between the capillary and the mitochondria. As oxygen is consumed during $\mathrm{HCA}$, the $\mathrm{PO}_{2}$ of the residual cranial blood drops, and hemoglobin will desaturate. Desaturation is not favorable during HCA because the affinity of hemoglobin for oxygen increases as temperature drops. At $20^{\circ} \mathrm{C}$ a hemoglobin saturation of $30 \%$ will be associated with a $\mathrm{PO}_{2}$ in the capillaries of 7 to 10 torr, and the capillary to mitochondria gradient will be abolished. Therefore, when the hemoglobin saturation approaches $30 \%$ during HCA, desaturation and oxygen delivery stops, thus causing hypoxia. Theoretically, the time limitation of $\mathrm{HCA}$ is due to the $\mathrm{PO}_{2}$ of the cerebral microcirculation and the duration necessary for the pressure gradient to become abolished. Monitoring cerebral oxygen saturation during HCA should permit the determination of the safe duration until the $\mathrm{PO}_{2}$ falls near $30 \%$ and hypoxia with neurologic damage ensues.

In an effort to prolong the safe duration of HCA, Ueda first described the use of RCP via the superior vena cava as a means of perfusing cerebral tissues during aortic arch reconstruction. This technique was a modification of the technique described by Mills and Ochsner ${ }^{14}$ to treat massive air embolism during CPB. Safi reported on 11 patients undergoing HCA with RCP in whom he attempted to use metabolic markers (creatine kinase BB) from blood obtained from the innominate and left common carotid arteries during HCA with RCP to assess cerebral ischemia. Unfortunately, the markers were inadequate because of rapid metabolic breakdown of creatine kinase $\mathrm{BB}$ in the blood. In addition, blood samples obtained from the innominate and common carotid arteries during RCP do not accurately reflect cerebral tissue saturation, because they also include blood that has perfused the cranium, soft tissues of the face, neck, and the right upper extremity.

To obtain a more accurate reflection of cerebral oxygen delivery with regard to metabolism and oxygen consumption during RCP, as well as to eliminate from the measurement blood from the cranium and soft tissues of the face, neck, and upper extremities, we used continuous spectroscopic measurement of cerebral hemoglobin oxygen saturation as the indicator of brain ischemia. Near-infrared light penetrates human tissue and is attenuated during transmission by scatter and absorption. The majority of attenuation in human cerebral tissue can be attributed to absorption by hemoglobin and oxyhemoglobin. ${ }^{14,21,22}$ Diffuse transmission spectroscopy involves the use of a single infrared point source and one or more optode receivers placed $1 \mathrm{~cm}$ or more from the source. ${ }^{13,23,24}$ In this arrangement, light propagates along a high probability course into the tissues of interest and then back to the surface. ${ }^{25,26}$ This process has the unique advantage of depth resolution, which allows cerebral hemoglobin to be measured independently of scalp and skull hemoglobin. ${ }^{8,20}$ Because oxyhemoglobin and hemoglobin have unique absorption profiles in the near-infrared spectrum, a tissue content ratio of the two can be determined and expressed as a percent hemoglobin oxygen saturation. Because $70 \%$ to $80 \%$ of the cerebral vascular tissue is venous, cerebral oximetry primarily represents cerebral venous saturation. ${ }^{13}$ Cerebral venous saturation is not an exact quantitation of cerebral capillary hemoglobin saturation but is actually a lower number, because it reflects the equilibration of both the blood being delivered retrogradely and the percent saturation after oxygen has migrated into the neuronal tissue. However, it will indicate an average saturation of the blood circulating in the brain. Theoretically, continuous measurement of cerebral oxygen saturation should be an excellent indicator of oxygen delivery to the neurons, because it affords an opportunity to know if the saturation nears $30 \%$, which would abolish the $\mathrm{PO}_{2}$ gradient and thus the delivery of oxygen. Therefore, using this noninvasive, continuous, and direct technique, one could monitor the safe duration of HCA with RCP by regulation of retrograde flow to keep the cerebral oxygen saturation greater than $30 \%$.

Because the cerebral oxygen saturation of patients supported by CPB is not widely known and has not been reported, each patient in our series acted as his or her own control during the procedure. We attempted to maintain cerebral oxygen saturation near pre-CPB levels ( $\pm 10 \%$ range) during $\mathrm{CPB}$ and $\mathrm{HCA}$ by regulation of RCP flow rates. Hemodilution may have a significant effect on baseline values according to infrared spectroscopy, and therefore it may be more correct 
to use a baseline value once CPB is established and balanced. Clearly, further controlled quantitative studies with infrared spectroscopy will be necessary to determine the usefulness of this technique in monitoring oxygen delivery for patients undergoing surgical procedures.

Our results demonstrate that $\mathrm{RCP}$ can prolong the 45-minute "safe" duration of HCA as defined by Svensson and lower the neurologic mortality and morbidity in this patient population, because we were able to extend our HCA times beyond 65 minutes in $12(34 \%)$ of our patients, with $11(92 \%)$ of these patients gaining meaningful consciousness within 24 hours and no significant gross motor or sensory deficit as noted by basic physical examination. This is in marked contrast to our previous experience, in which all three of our patients, who had a normal preoperative neurologic history and physical examination and, who underwent aortic arch repair with HCA without RCP for greater than 65 minutes, never regained meaningful consciousness and died. We are not proposing that the patients in this series are without any neurologic morbidity, because no sensitive measurements of neuropsychometric function were performed. We are presently involved in a prospective study with members of the neuroscience department using standardized quantitative tests to compare preoperative and postoperative neuropsychometric performance of patients undergoing operations with HCA and RCP, as well as patients undergoing aortic operations without HCA and RCP, patients undergoing CPB for operations other than aortic repair, and a last group undergoing thoracic operations without $\mathrm{CPB}$. We are uncomfortable including a group undergoing surgery with HCA without RCP because of our previous poor results in this patient population.

HCA has been an ally with a double-edged sword for the thoracic surgeon. It affords the means to complete this type of complicated operation, but the cost is a limited time for ischemic protection, a significant coagulopathy for the patient, and a prolonged operative time because of cooling and rewarming the patient. RCP in association with HCA in our series allowed for a prolongation of the ischemic period to provide the time necessary to accomplish a precise and complete surgical repair. Controlled clinical trials are necessary to characterize the mechanism, extent, and time limitation of ischemic protection and the detrimental effects from this modality of therapy. Whether monitoring the cerebral oxygen saturation is of clinical significance or just a security blanket for the surgeon is totally unclear and will need delineation and verification in the laboratory and in good controlled clinical studies.

\section{Conclusion}

RCP appears to significantly extend the safe duration for HCA for preservation of meaningful consciousness and gross motor and sensory function. Cerebral oximetry is a continuous, noninvasive, and direct method of determining cerebral oxygen saturation and may be a beneficial tool to monitor the efficacy of RCP during HCA. Further controlled quantitative studies are indicated. RCP can be safely administered through either a median sternotomy or a posterolateral approach as described.

\section{REFERENCES}

1. DeBakey ME, Crawford ES, Cooley DA, Morris GC. Successful resection of fusiform aneurysm of aortic arch with replacement of homograft. Surg Gynecol Obstet 1957;105:657.

2. DeBakey ME, Henly WS, Cooley DA, Crawford ES, Morris GC, Beall AC. Aneurysms of the aortic arch factors influencing operative risk. Surg Clin North Am 1962;42:1543.

3. Bloodwell RD, Hallman GL, Cooley DA. Total replacement of the aortic arch and the "subclavian steal" phenomenon. Ann Thorac Surg 1968;5:236.

4. Griepp RB, Stinson EB, Hollingsworth JF, Buehler D. Prosthetic replacement of the aortic arch. J THORaC Cardiovasc Surg 1975;70:1051-63.

5. Crawford ES, Saleh SA. Transverse aortic arch aneurysm: improved results of treatment employing new modifications of aortic reconstruction and hypothermic cerebral circulatory arrest. Ann Surg 1981;194:180-8.

6. Crawford ES, Svensson LG, Coselli JS, Safi HJ, Hess KR. Surgical treatment of aneurysm and/or dissection of the ascending aorta, transverse aortic arch, and ascending aorta and transverse aortic arch: factors influencing survival in 717 patients. J THORAC CARDIOVASC SURG 1989;98:659-74.

7. Svensson LG, Crawford ES, Coselli JS, Safi HJ, Hess $\mathrm{KR}$. The impact of cardiovascular operation on survival in the Marfan patient. Circulation 1989;80 (Suppl):I232-42.

8. Svensson LG, Crawford ES, Hess DR, Coselli JS, Safi HJ. Dissection of the aorta and dissecting aortic aneurysms: improving early and long-term surgical results. Circulation 1990;82(Suppl):IV24-48.

9. Borst HG, Walterbusch G, Schaps D. Extensive aortic replacement using "elephant trunk" prosthesis. Thorac Cardiovasc Surg 1983;31:37-40.

10. Svensson LG, Crawford ES, Hess KR, et al. Deep hypothermia with circulatory arrest: determinants of 
stroke and early mortality in 656 patients. J Cardiovasc Surg 1993;106:19-31.

11. Ueda Y, Miki S, Kusuhara K, Okita Y, Tahata T, Yamanaka K. Surgical treatment of aneurysm or dissection involving the ascending aorta and aortic arch, utilizing circulatory arrest and retrograde cerebral perfusion. J Cardiovasc Surg 1990;31:553-8.

12. Safi HJ, Brien HW, Winter JN, et al. Protection via cerebral retrograde perfusion during aortic arch aneurysm repair. Ann Thorac Surg 1993;56:270-6.

13. McCormick PW, Stewart M, Goetting MG, et al. Noninvasive cerebral optical spectroscopy for monitoring cerebral oxygen delivery and hemodynamics. Crit Care Med 1991;19:89-97.

14. Mills NL, Ochsner JL. Massive air embolism during cardiopulmonary bypass. J THORAC CARdiovasc SURG 1980;80:708-17.

15. Aoyagi M, Flasterstein AH, Barnette J, et al. Cerebral effects of profound hypothermia (18 degrees CP and circulatory arrest. Circulation 1975;52:(Suppl):I52-60.

16. Astrup J, Skovsted P, Gjerris F, et al. Increase in extracellular potassium in the brain during circulatory arrest: effects of hypothermia, lidocaine, and thiopental. Anesthesiology 1981;55:256-62.

17. Mchedlishvili G. Cerebral arterial behavior providing constant cerebral blood flow, pressure, and volume. In: Bevan JA, ed. Arterial behavior and blood circulation in the brain. New York: Plenum Press, 1986:294-302.

18. Stern MD. In vivo evaluation of microcirculation by coherent light scattering. Nature 1975;254:56-8.

19. Takatani S, Cheung PW, Ernst EA. A noninvasive tissue reflectance oximeter: an instrument for measurement of tissue hemoglobin oxygen saturation in vivo. Ann Biomed Eng 1980;8:1-15.

20. Ausman JI, McCormick PW, Stewart M. Cerebral oxygen metabolism during hypothermic circulatory arrest in humans. J Neurosurg 1993;79:810-15.

21. Hazeki O, Tamura M. Quantitative analysis of hemoglobin oxygenation state of rat brain in situ by nearinfrared spectrophotometry. J Appl Physiol 1988;64: 796-802.

22. Willford DC, Hill EP, Moores WY. Theoretical analysis of oxygen transport during hypothermia. J Clin Monit 1986;2:30-43.

23. McCormick PW, Stewart M, Goetting MG, et al. Regional cerebrovascular oxygen saturation measured by optical spectroscopy in humans. Stroke 1991; 22:596-602.

24. McCormick PW, Stewart M, Lewis G. Noninvasive measurement of regional cerebrovascular oxygen saturation in humans using optical spectroscopy: time resolved spectroscopy and imaging of tissues. Proceedings of the Society of Proto-optical Instrumentation Engineers. The International Society for Optical Engineering. 1991;1431:294-302.
25. Nossa R, Bonner RF, Weiss GH. Influence of path length on remote optical sensing of properties of biological tissue. Appl Opt 1989;28:2238-44.

26. Patterson MS, Chance B, Wilson BC. Time resolved reflectance and transmittance for the noninvasive measurement of tissue optical properties. Appl Opt 1989;28:2331-2336.

\section{Discussion}

Dr. Thomas Z. Lajos (Buffalo, N.Y.). I would like to make a comment and ask a question.

We have been using an arteriovenous bridge at Buffalo General Hospital for more than 25 years. It is located off the operating table at the perfusionist's level, and it is interspaced between the arterial and venous lines of the pump circuit. This "bridge" is extremely useful in different situations. During routine perfusion this bridge is clamped. If the patient has bicaval cannulation, we can deliver through this bridge retrograde (cold arterial blood) perfusion during circulatory arrest just by changing the clamp and reapplying it on the arterial line distally.

We can monitor the volume and the pressure as well. We have used this system on more than 15 patients in The Buffalo General Hospital in the past 2 to 3 years. This is a very simple system; we do not have to put an extra superior vena cava cannula in, and other usages of this bridge across the arteriovenous lines enable us to perform other maneuvers in different situations: for example, pump failure, retrograde venous perfusion, and debubbling. ${ }^{\text {. }}$

My question is this: You have elegantly monitored the oxygen delivery to the brain of the patient, but how did you monitor the possibility of an impending "oxygen lack" or damage of the central nervous system? Was there any electroencephalographic (EEG) or some other method to monitor the central nervous system status? With blood samples, we have also proved that oxygen is extracted and lactic acidosis does not ensue, but we have failed to find a sensitive method of monitoring its physiologic status.

\section{REFERENCE}

1. Diesfeld T, Lajos TZ, Schimert G. Arterial venous bridge on cardiopulmonary bypass. J Card Surg 1994; 9:128-30.

Dr. Deeb. Thank you for your comments. We initially used the EEG to monitor our patients, but when the patient's temperature fell below $19^{\circ} \mathrm{C}$, our EEGs went flat line. Thus the entire time that we were having HCA the EEG was flat line and was of no help to us in monitoring cerebral function.

As far as using somatosensory and other evoked potentials, I believe that by the time we saw changes, a lot of irreversible damage would have occurred. I do not know how these techniques would help me change what I do during the operation, so we used cerebral oxygen saturation monitoring as our technique. Our premise was that ischemia/hypoxia caused injury. If we monitored our patients' oxygen delivery and regulated our flow to avoid hypoxia, then we would avoid neurologic deficit. 